\title{
Relationships Between Level and Change in Sarcopenia and Other Body Composition Components and Adverse Health Outcomes: Findings from the Health, Aging, and Body Composition Study
}

\author{
Leo D. Westbury ${ }^{1}$ - Holly E. Syddall ${ }^{1}$ - Nicholas R. Fuggle ${ }^{1}$ - Elaine M. Dennison ${ }^{1,2}$ - Nicholas C. Harvey ${ }^{1,3}$. \\ Jane A. Cauley ${ }^{4}$. Eric J. Shiroma ${ }^{5} \cdot$ Roger A. Fielding $^{6} \cdot$ Anne B. Newman $^{4} \cdot$ Cyrus Cooper $^{1,3,7}$
}

Received: 19 August 2020 / Accepted: 27 October 2020 / Published online: 15 November 2020

(c) The Author(s) 2020

\begin{abstract}
We investigated how baseline values and rates of decline in components of sarcopenia and other body composition parameters relate to adverse clinical outcomes using the Health, Aging, and Body Composition Study. 2689 participants aged 70-79 years were studied. Appendicular lean mass, whole body fat mass, and total hip BMD were ascertained using DXA; muscle strength by grip dynamometry; and muscle function by gait speed. Baseline values and 2-3 year conditional changes (independent of baseline) in each characteristic were examined as predictors of mortality, hospital admission, low trauma fracture, and recurrent falls in the subsequent 10-14 years using Cox regression (generalized estimating equations used for recurrent falls) with adjustment for sex, ethnicity, age, and potential confounders. Lower levels and greater declines in all parameters (excluding hip BMD level) were associated $(p<0.05)$ with increased rates of mortality; fully-adjusted hazard ratios per SD lower gait speed and grip strength were 1.27 (95\% CI 1.19, 1.36) and $1.14(1.07,1.21)$, respectively. Risk factors of hospital admission included lower levels and greater declines in gait speed and grip strength, and greater declines in hip BMD. Lower levels and greater declines in fat mass and hip BMD were associated with low trauma fracture. Lower gait speed, higher fat mass, and both lower levels and greater declines in grip strength were related to recurrent falls. Lower baseline levels and greater declines in musculoskeletal parameters were related to adverse outcomes. Interventions to maximize peak levels in earlier life and reduce rates of age-related decline may reduce the burden of disease in this age group.
\end{abstract}

Keywords Epidemiology $\cdot$ Sarcopenia $\cdot$ Osteoporosis $\cdot$ Aging $\cdot$ Older people

\section{Introduction}

Sarcopenia, the accelerated loss of skeletal muscle mass and strength with age, is associated with physical disability, mortality, and significant healthcare expenditure [1]. Although

Electronic supplementary material The online version of this article (https://doi.org/10.1007/s00223-020-00775-3) contains supplementary material, which is available to authorized users.

Cyrus Cooper

cc@mrc.soton.ac.uk

1 MRC Lifecourse Epidemiology Unit, University of Southampton, Southampton, UK

2 Victoria University of Wellington, Wellington, New Zealand

3 NIHR Southampton Biomedical Research Centre, University of Southampton and University Hospital Southampton NHS Foundation Trust, Southampton, UK

4 Department of Epidemiology, Graduate School of Public Health, University of Pittsburgh, Pittsburgh, USA there is no consensus algorithm for diagnosing sarcopenia,

5 Laboratory of Epidemiology and Population Sciences, Intramural Research Program, National Institute on Aging, Baltimore, USA

6 Nutrition, Exercise Physiology, and Sarcopenia Laboratory, Jean Mayer USDA Human Nutrition Research Center on Aging, Tufts University, Boston, USA

7 NIHR Oxford Biomedical Research Centre, University of Oxford, Oxford, UK 
the recent convergence in definitions of sarcopenia recognizes muscle mass (appendicular lean mass), strength (grip strength), and function (gait speed) as key components [2, 3].

Previous research has examined levels and changes in some sarcopenia components in relation to adverse health outcomes. For example, the relationship between lower levels and greater declines in gait speed and grip strength in relation to greater risk of mortality has been reported previously [4-6]. However, there is uncertainty with regard to the strength of associations between baseline values and subsequent rates of decline in these measures in relation to clinical outcomes in older people. Furthermore, to our knowledge, no studies have explored both baseline values and changes in sarcopenia components in relation to multiple adverse health outcomes among a single cohort of community-dwelling older people.

Many cohort studies have suggested four critical adverse health outcomes associated with sarcopenia in older people: death (Third National Health and Nutrition Examination Survey [7] and InCHIANTI Study [8]), hospitalization (InCHIANTI Study [8]), fracture (Osteoporotic Fractures in Men (MrOS) Study [9]), and falls (Healthy Ageing Initiative Cohort Study [10]). Accordingly, in this paper, we examine baseline values and changes in sarcopenia components in relation to these outcomes among participants in the Health, Aging, and Body Composition (Health ABC) Study, USA. For completeness, levels and changes in other aspects of body composition, namely fat mass and bone mineral density, are also included as predictors.

\section{Methods}

\section{The Health, Aging, and Body Composition Study}

The Health ABC Study comprises 3075 US men and women (aged 70-79 years at baseline) who were recruited in 1997-1998 [11]. A random sample of white and all the black Medicare beneficiaries from around Memphis (Tennessee) and Pittsburgh (Pennsylvania) was obtained. Sampled participants received a mailing followed by a telephone eligibility screen. The original purpose of the Health ABC Study was to understand risk factors for the decline of function and change in body composition among healthy older people [12]. Therefore, the cohort was selected to be free of mobility limitation at baseline. Individuals reporting no difficulty in walking one quarter of a mile or climbing 10 stairs were considered eligible. Individuals with the following characteristics were excluded: inability to communicate with the interviewer; clear cognitive impairment; having a life-threatening illness or difficulties with activities of daily living (ADL); requiring a walking aid; having an intention of moving outside the area within three years; or currently enrolled in a lifestyle intervention trial. Written informed consent was provided by all participants and the study was approved by the institutional review boards at the University of Tennessee and the University of Pittsburgh. Initially, participant information was ascertained from annual examinations from baseline (Year 1: 1997-1998) to Year 6 (2002-2003) and from biannual questionnaires from baseline to Year 14 (2010-2011).

\section{Ascertainment of Sarcopenia and Other Body Composition Parameters}

Gait speed was ascertained at Years 2 (1998-1999) and 4 (2000-2001) by asking participants to walk at their normal speed down a corridor over a total distance of $20 \mathrm{~m}$. Grip strength was measured twice for each hand at Years 1 and 4 using a Jamar hydraulic dynamometer with the participant in the sitting position with the arm to be tested resting on the table and the elbow held at approximately a right angle, according to a standardized protocol [13]; the calibration of the dynamometers was checked regularly. Maximum grip strength at each time point was used for analyses. Grip strength values were set to missing for participants with severe hand pain or recent surgery. Whole body dual-energy X-ray absorptiometry scans (Hologic QDR 4500A; Hologic, Bedford, MA, USA) were performed at Years 1 and 4 and used to ascertain whole body fat and appendicular lean mass (ALM). Total hip BMD was measured using the same device at Years 1 and 3 (1999-2000). The reproducibility and validity of this scanner have been previously reported [14, 15]. Regular DXA phantom scans were performed for quality control and calibration purposes.

\section{Ascertainment and Derivation of Potential Confounders}

The study methodology has been described in detail previously [12]. In brief, at baseline (Year 1), sex, ethnicity, educational attainment, home ownership, and health behaviors such as smoking status and alcohol consumption were self-reported using questionnaires. Height and weight were measured using a Harpenden Stadiometer (Holtain Ltd, Crosswell, UK) and a standard balance beam scale, respectively. Height and weight were highly correlated $(r=0.45$, $p<0.001$ for men; $r=0.31, p<0.001$ for women); to avoid multi-collinearity in models, a sex-specific standardized residual of weight-adjusted-for-height was derived as a measure of adiposity. Physical activity over the past 7 days was assessed using an interviewer-administered questionnaire. Approximate metabolic equivalent unit values were assigned to reported activities and intensity levels to derive caloric expenditure in $\mathrm{kcal} / \mathrm{kg} / \mathrm{h}$ [16]. Total kilocalories 
expended per week was calculated by multiplying the participant's weight $(\mathrm{kg})$ by the sum of the caloric expenditure for all activities performed, as previously described [17]. As in previous analyses $[18,19]$, the number of self-reported comorbidities (ever told by a doctor) was used as a marker of comorbidity out of the following: stroke, diabetes, Parkinson's disease, chronic obstructive pulmonary disease, heart attack or myocardial infarction, congestive heart failure and hypertension. Cognitive function was measured using the Modified Mini-Mental State Examination (3MS) with scores ranging from 0 to 100 (higher scores indicate better function) [20]; scores were dichotomized for analysis; and participants with scores $<80$ were regarded as having low cognitive function [21].

At Year 2, dietary intake over the previous year was assessed using a nurse-administered food frequency questionnaire (FFQ) comprising 108 items. To assess the extent to which Health $\mathrm{ABC}$ participants' diets conformed to recommendations of the Dietary Guidelines for Americans of 1995 and the Food Guide Pyramid of 1992, a healthy eating index (HEI), ranging from 0 to 100 , was calculated for each participant; higher scores reflected healthier diets [22].

\section{Ascertainment of Adverse Outcomes}

Deaths from baseline until 30th September 2014 were determined from death certificates, hospital records, and interviews with next of kin. All deaths were adjudicated by a central committee. Participants reported hospital admissions during follow-up and were asked specific questions about their admissions every 6 months [23]. Medical records for each reported admission were collected; these contained information on admission and discharge dates and the main reason for admission. Information on diagnoses and length of stay were checked by local review. Fractures were ascertained by self-report every 6 months and confirmed by radiology reports. For this analysis, fracture events were limited to low trauma fractures, defined as 'spontaneous or with modest trauma, such as a fall from a standing height' [24]. Adjudication for admissions and fractures was complete until 14th August 2012; events occurring after this date were not used for this analysis. At every year of follow-up, up to and including Year 14 (2010-2011), participants reported the number of times they had fallen over and landed on the floor or the ground during the last 12 months; an indicator variable for recurrent falls $(\geq 2)$ was derived at each year of follow-up.

\section{Derivation of Exposures}

For gait speed, grip strength, ALM, fat mass, and hip BMD, exposures were baseline levels at Year 1 (Year 2 for gait speed) and conditional changes from Years 1 to 4 (2 to 4 for gait speed and 1 to 3 for hip BMD). Conditional changes (independent of baseline) were characterized by residuals from sex-specific linear regression models for parameters at follow-up on baseline parameters with adjustment for individual follow-up duration.

\section{Statistical Methods}

Baseline participant characteristics were described using means and standard deviations (SD), medians and interquartile ranges and frequency and percentage distributions. For each parameter, outcomes only included adverse events (deaths, hospital admissions, low trauma fractures, and recurrent falls) occurring after Year 4 (Year 3 for hip BMD). Exposures were examined in relation to death, hospital admission, and low trauma fracture using time-to-first-event Cox proportional hazards models with death as a censoring event for the latter two. For those who did not experience the adverse event and did not leave the study early, time at risk ended on 30th September 2014 for death and 14th August 2012 for hospital admission and low trauma fracture. Exposures in relation to risk of recurrent falls were examined using a generalized estimating equations (GEE) model, as in previous studies [25-27], with a binomial distribution, logit link function, and robust standard errors to account for clustering within individuals.

Adjustments were selected a priori and included measures of anthropometric, sociodemographic, lifestyle, and clinical characteristics that are known to influence both the sarcopenia and other body composition components [28] and risk of the adverse health outcomes considered. All models were adjusted for baseline age and for a four-level variable reflecting the possible combinations of sex and ethnicity; there was no evidence of interactions between exposures and this four-level variable. Fully-adjusted models also accounted for height, weight-for-height residual (not used in models for level relating to ALM and fat mass due to collinearity), smoking status (ever vs never), alcohol consumption, healthy eating index, physical activity, educational attainment, home ownership, cognitive function, and number of comorbidities. Cox models for hospital admission and low trauma fracture were also stratified on whether or not participants experienced these events before the start of the survival analysis follow-up (Year 3 for hip BMD and Year 4 for the remaining parameters); similarly, GEE models for recurrent falls were adjusted for previous recurrent falls.

All analyses were based on the sample of 2689 participants with data on both baseline level and conditional change for at least one of the parameters (gait speed, grip strength, ALM, fat mass, and hip BMD). Exposures (baseline levels and conditional changes in parameters) were standardized (sex-specific) in models to enable the 
comparison of effect sizes. Analyses were conducted using Stata, release 15 (StataCorp, College Station, TX, USA).

Several sensitivity analyses were performed. The number of hospital admissions was analyzed using negative binomial regression with robust variance estimation as in a previous study [23]; a competing risk analysis for hospital admission and low trauma fracture was implemented, with death as a competing event, using the Fine-Gray subdistribution hazards model [29]; and cause-specific mortality (cardiovascular-related, cancer-related, and other) was examined as an outcome. Further sensitivity analyses involved examining: ALM residuals (derived from sexspecific models predicting ALM from height and fat mass as in previous analyses [30]) which reflect whether levels of ALM were higher or lower than expected, given stature and fat mass; and changes in ALM after adjusting for changes in weight as in previous analyses [31] which reflect whether declines in ALM were greater than expected, given total weight change.

\section{Results}

\section{Descriptive Statistics}

Baseline participant characteristics among the analysis sample of 2689 Health ABC participants according to sex and ethnicity are presented in Table 1 . Mean and standard deviation (SD) for age was 74.1 (2.8) years. Women had higher fat mass, but all other components of sarcopenia and additional body composition parameters were greater among men ( $p<0.001$ for all associations). Survival analysis descriptive statistics are presented in Table 2 for the 2480 participants with data on exposures and survival analysis follow-up times starting at Year 4; these statistics differ for hip BMD where follow-up time started at Year 3. Median follow-up time (number of years from Year 4 to the first event or until participants were censored) was greater for death (11.3) and low trauma fracture (9.8) compared with hospital admission (3.4). A significantly $(p<0.05)$ higher

Table 1 Baseline participant characteristics according to sex and ethnicity

\begin{tabular}{|c|c|c|c|c|c|c|}
\hline \multirow{2}{*}{$\begin{array}{l}\text { Characteristic [mean (SD) or } \\
N(\%)]\end{array}$} & \multicolumn{3}{|l|}{ Men } & \multicolumn{3}{|l|}{ Women } \\
\hline & White $(n=846)$ & Black $(n=446)$ & All $(n=1292)$ & White $(n=782)$ & Black $(n=615)$ & All $(n=1397)$ \\
\hline Age (years) & $74.3(2.9)$ & $74.1(2.8)$ & $74.2(2.8)$ & $74.0(2.8)$ & $73.8(2.9)$ & $73.9(2.9)^{\dagger}$ \\
\hline Height (m) & $1.73(0.06)$ & $1.73(0.07)$ & $1.73(0.07)$ & $1.60(0.06)$ & $1.60(0.06)$ & $1.60(0.06)$ \\
\hline Weight (kg) & $81.5(12.4)$ & $81.7(14.3)$ & $81.6(13.1)$ & $66.2(12.1)$ & $75.7(15.8)$ & $70.4(14.6)^{\dagger}$ \\
\hline BMI $\left(\mathrm{kg} / \mathrm{m}^{2}\right)$ & $27.0(3.7)$ & $27.2(4.3)$ & $27.1(3.9)$ & $26.0(4.5)$ & $29.7(5.9)$ & $27.6(5.4)^{\dagger}$ \\
\hline Ever smoked & $592(70.1 \%)$ & $306(68.6 \%)$ & $898(69.6 \%)$ & $316(40.4 \%)$ & $269(43.8 \%)$ & $585(41.9 \%)$ \\
\hline Current drinker & $544(64.6 \%)$ & $205(46.3 \%)$ & $749(58.3 \%)^{\dagger}$ & $415(53.2 \%)$ & $191(31.1 \%)$ & $606(43.5 \%)^{\dagger}$ \\
\hline Physical activity (Mcal/week) & $5.7(3.2,8.9)$ & $4.7(2.4,9.1)$ & $5.4(3.0,8.9)$ & $4.6(2.8,7.3)$ & $4.6(2.6,7.6)$ & $4.6(2.7,7.5)$ \\
\hline Healthy eating index ${ }^{a}$ & $70.6(11.5)$ & $63.7(12.1)$ & $68.3(12.1)^{\dagger}$ & $72.6(11.7)$ & $68.5(11.8)$ & $70.8(11.9)^{\dagger}$ \\
\hline Post-secondary education & $507(60.0 \%)$ & $120(27.0 \%)$ & $627(48.6 \%)^{\dagger}$ & $377(48.3 \%)$ & $170(27.8 \%)$ & $547(39.3 \%)^{\dagger}$ \\
\hline $\begin{array}{l}\text { Home ownership (does not } \\
\text { own) }\end{array}$ & $147(17.8 \%)$ & $111(25.1 \%)$ & $258(20.3 \%)^{\dagger}$ & $205(26.8 \%)$ & $220(36.3 \%)$ & $425(31.0 \%)^{\dagger}$ \\
\hline \multicolumn{7}{|l|}{ Number of comorbidities ${ }^{\mathrm{b}}$ : } \\
\hline 0 & $363(45.0 \%)$ & $141(33.5 \%)$ & $504(41.0 \%)^{\dagger}$ & $382(50.5 \%)$ & $154(26.6 \%)$ & $536(40.1 \%)^{\dagger}$ \\
\hline 1 & $305(37.8 \%)$ & $170(40.4 \%)$ & $475(38.7 \%)^{\dagger}$ & $306(40.5 \%)$ & $290(50.0 \%)$ & $596(44.6 \%)^{\dagger}$ \\
\hline 2 & $120(14.9 \%)$ & $92(21.9 \%)$ & $212(17.3 \%)^{\dagger}$ & $55(7.3 \%)$ & $110(19.0 \%)$ & $165(12.4 \%)^{\dagger}$ \\
\hline $3 / 4$ & $19(2.4 \%)$ & $18(4.3 \%)$ & $37(3.0 \%)^{\dagger}$ & $13(1.7 \%)$ & $26(4.5 \%)$ & $39(2.9 \%)^{\dagger}$ \\
\hline Cognitive function (3MS score) & $94.0(90.0,97.0)$ & $87.0(79.5,92.5)$ & $92.0(86.0,96.0)^{\dagger}$ & $95.0(92.0,97.0)$ & $89.0(83.0,94.0)$ & $93.0(88.0,96.0)^{\dagger}$ \\
\hline Gait speed $(\mathrm{m} / \mathrm{s})^{\mathrm{a}}$ & $1.24(0.19)$ & $1.10(0.20)$ & $1.19(0.20)^{\dagger}$ & $1.16(0.19)$ & $1.02(0.19)$ & $1.10(0.20)^{\dagger}$ \\
\hline Grip strength (kg) & $39.8(7.7)$ & $43.0(8.5)$ & $40.9(8.1)^{\dagger}$ & $23.7(5.1)$ & $26.6(6.3)$ & $25.0(5.8)^{\dagger}$ \\
\hline $\operatorname{ALM}(\mathrm{kg})$ & $23.3(3.2)$ & $25.1(3.9)$ & $23.9(3.5)^{\dagger}$ & $15.3(2.4)$ & $18.3(3.2)$ & $16.6(3.1)^{\dagger}$ \\
\hline Fat mass (kg) & $24.8(6.9)$ & $23.4(7.3)$ & $24.3(7.1)^{\dagger}$ & $27.1(7.9)$ & $31.6(10.1)$ & $29.1(9.3)^{\dagger}$ \\
\hline Hip BMD $\left(\mathrm{g} / \mathrm{cm}^{2}\right)$ & $0.95(0.14)$ & $1.02(0.15)$ & $0.97(0.15)^{\dagger}$ & $0.77(0.13)$ & $0.86(0.15)$ & $0.81(0.15)^{\dagger}$ \\
\hline
\end{tabular}

$S D$ standard deviation, $A L M$ appendicular lean mass, $B M D$ bone mineral density, $3 M S$ modified mini-mental state examination

${ }^{\dagger}$ Statistically significant ethnic differences within sex $(p<0.05)$; differences between sexes were significant $(p<0.05)$ for all characteristics

${ }^{a}$ Ascertained at year 2

${ }^{b}$ Median (lower quartile, upper quartile) number of the following comorbidities (ever told by doctor): stroke, diabetes, Parkinson's disease, chronic obstructive pulmonary disease, heart attack or myocardial infarction, congestive heart failure, and hypertension 
Table 2 Descriptive statistics for analysis of adverse health outcomes

\begin{tabular}{|c|c|c|c|c|c|c|}
\hline \multirow{2}{*}{$\begin{array}{l}\text { Characteristic [mean (SD) or } N \\
(\%)]\end{array}$} & \multicolumn{3}{|l|}{ Men } & \multicolumn{3}{|l|}{ Women } \\
\hline & White $(n=789)$ & Black $(n=398)$ & All $(n=1187)$ & White $(n=732)$ & Black $(n=561)$ & All $(n=1293)$ \\
\hline \multicolumn{7}{|l|}{ Death } \\
\hline Incidence & $510(64.6 \%)$ & $282(70.9 \%)$ & $792(66.7 \%)^{* \dagger}$ & $387(52.9 \%)$ & $328(58.5 \%)$ & $715(55.3 \%)^{* \dagger}$ \\
\hline Follow-up time (years) & $10.6(6.5,13.5)$ & $9.2(4.5,13.3)$ & $10.2(5.8,13.4)^{* \dagger}$ & $12.4(8.6,13.6)$ & $11.4(7.1,13.5)$ & $12.1(7.9,13.6)^{* \dagger}$ \\
\hline \multicolumn{7}{|l|}{ Hospital admission } \\
\hline Incidence & $695(88.1 \%)$ & $345(86.7 \%)$ & $1040(87.6 \%)^{*}$ & $617(84.3 \%)$ & $476(84.8 \%)$ & $1093(84.5 \%)^{*}$ \\
\hline Follow-up time (years) & $3.0(1.2,6.3)$ & $2.9(1.2,5.4)$ & $2.9(1.2,6.0)^{*}$ & $4.3(1.8,8.4)$ & $3.4(1.3,7.5)$ & $3.9(1.6,8.1)^{* \dagger}$ \\
\hline Prevalence before follow-up & $305(38.7 \%)$ & $154(38.7 \%)$ & $459(38.7 \%)^{*}$ & $205(28.0 \%)$ & $204(36.4 \%)$ & $409(31.6 \%)^{* \dagger}$ \\
\hline \multicolumn{7}{|l|}{ Low trauma fracture } \\
\hline Incidence & $106(13.7 \%)$ & $23(5.8 \%)$ & $129(11.0 \%)^{* \dagger}$ & $209(29.8 \%)$ & $63(11.6 \%)$ & $272(21.9 \%)^{* \dagger}$ \\
\hline Follow-up time (years) & $9.8(5.7,11.5)$ & $8.7(4.3,11.4)$ & $9.6(5.2,11.5)^{\dagger}$ & $10.0(5.0,11.6)$ & $10.2(5.6,11.6)$ & $10.1(5.2,11.6)$ \\
\hline Prevalence before follow-up & $16(2.1 \%)$ & $3(0.8 \%)$ & $19(1.6 \%)^{*}$ & $42(6.0 \%)$ & $14(2.6 \%)$ & $56(4.5 \%)^{*, \dagger}$ \\
\hline \multicolumn{7}{|l|}{ Recurrent falls } \\
\hline Incidence & $347(45.6 \%)$ & $124(33.5 \%)$ & $471(41.6 \%)^{*, \dagger}$ & $342(47.8 \%)$ & $239(44.1 \%)$ & $581(46.2 \%)^{*}$ \\
\hline Prevalence before follow-up & $162(21.3 \%)$ & $61(16.5 \%)$ & $223(19.7 \%)$ & $147(20.5 \%)$ & $115(21.2 \%)$ & $262(20.8 \%)$ \\
\hline
\end{tabular}

Of the entire study sample, 265/3075 (8.6\%) died before Year 4. $256(63.8 \%)$ of those who had a low trauma fracture also died during follow-up; figures for hospital admission and recurrent falls were 1369 (64.2\%) and $662(62.9 \%)$. Statistics presented for follow-up time starting at Year 4 among 2480 individuals with level and change measures for at least one of the following parameters: gait speed, grip strength, ALM, and fat mass; these statistics differ for hip BMD as the exposure where follow-up time started at Year 3. Individuals with no recurrent falls and missing responses for recurrent falls at Year 4 and after $(n=713)$ were regarded as not having had recurrent falls for these descriptive statistics as these individuals were included in generalized estimating equations models for recurrent falls (their non-missing responses contribute information)

* Statistically significant sex differences $(p<0.05)$

${ }^{\dagger}$ Statistically significant ethnic differences within $\operatorname{sex}(p<0.05)$

proportion of men than women died (66.7\% vs 55.3\%) or had a hospital admission ( $87.6 \%$ vs $84.5 \%$ ); a higher proportion of women than men had low trauma fractures $(21.9 \%$ vs $11.0 \%$ ) or recurrent falls ( $46.2 \%$ vs $41.6 \%$ ).

Compared to the 386 participants who were not included in the analytical sample, both men and women in the analytical sample had fewer comorbidities at baseline and were more likely to be white, have post-secondary education and to owner occupy their home ( $p<0.05$ for all associations).

\section{Baseline Values and Change in Sarcopenia and Other Body Composition Parameters and Risk of Adverse Outcomes}

The risk of each adverse outcome per SD lower baseline value and per SD greater decline in each parameter is presented in Tables 3 and 4, respectively. Figure 1 illustrates these associations for components of sarcopenia (gait speed, grip strength and ALM), and Fig. 2 illustrates these associations for body composition parameters (ALM, fat mass and hip BMD).

Lower baseline values and greater declines in all parameters (excluding the fully-adjusted association for hip BMD level) were associated with increased rates of mortality. For example, fully-adjusted hazard ratios $(95 \%$ CI) for mortality per SD lower level of gait speed, grip strength and ALM were 1.27 (1.19, 1.36), 1.14 (1.07, $1.21)$, and $1.17(1.08,1.26)$, respectively; corresponding estimates per SD greater decline in these parameters were $1.19(1.12,1.26), 1.09(1.03,1.16)$, and 1.15 $(1.08,1.22)$. Risk factors of hospital admission included lower levels and greater declines in gait speed and grip strength, and greater declines in hip BMD. Lower levels and greater declines in fat mass and hip BMD were related to increased risk of low trauma fracture. Lower levels of gait speed, higher levels of fat mass, and both lower levels and greater declines in grip strength were associated with greater risk of recurrent falls. All these associations were robust after adjustment for sex, ethnicity, and age and in fully-adjusted analysis.

\section{Relative Contribution of Baseline Values and Change in Sarcopenia Components to Rates of Mortality}

Of the variation in mortality explained by a model including grip strength level and change as predictors, grip strength level explained around $50 \%$ of this variation; corresponding figures for gait speed and ALM level were 70\% and $20 \%$, respectively. 
Table 3 Muscle function, body composition, and adverse outcomes: impact of baseline indices

\begin{tabular}{|c|c|c|c|c|c|c|c|c|c|}
\hline \multirow[t]{2}{*}{ Predictor } & \multirow[t]{2}{*}{ Model } & \multicolumn{2}{|l|}{ Death } & \multicolumn{2}{|c|}{ Hospital admission } & \multicolumn{2}{|c|}{ Low trauma fracture } & \multicolumn{2}{|l|}{ Recurrent fall } \\
\hline & & $\% \mathrm{CI})$ & $p$-value & $5 \% \mathrm{CI})$ & $p$-value & $\operatorname{HR}(95 \% \mathrm{CI})$ & $p$-value & OR $(95 \%$ CI $)$ & $p$-value \\
\hline \multirow[t]{2}{*}{ Gait speed } & 1 & $1.31(1.24,1.39)$ & $<0.001$ & $1.18(1.12,1.24)$ & $<0.001$ & $1.12(1.00,1.25)$ & 0.059 & $1.18(1.09,1.27)$ & $<0.001$ \\
\hline & 2 & $1.27(1.19,1.36)$ & $<0.001$ & $1.14(1.08,1.21)$ & $<0.001$ & $1.12(0.99,1.27)$ & 0.070 & $1.16(1.06,1.27)$ & 0.001 \\
\hline \multirow[t]{2}{*}{ Grip strength } & 1 & $1.13(1.07,1.20)$ & $<0.001$ & $1.09(1.04,1.14)$ & $<0.001$ & $1.18(1.05,1.31)$ & 0.004 & $1.14(1.04,1.24)$ & 0.003 \\
\hline & 2 & $1.14(1.07,1.21)$ & $<0.001$ & $1.13(1.07,1.19)$ & $<0.001$ & $1.13(0.99,1.29)$ & 0.061 & $1.13(1.03,1.24)$ & 0.012 \\
\hline \multirow[t]{2}{*}{ ALM } & 1 & $1.07(1.01,1.14)$ & 0.018 & $0.99(0.95,1.04)$ & 0.807 & $1.11(0.99,1.26)$ & 0.084 & $0.94(0.87,1.02)$ & 0.117 \\
\hline & 2 & $1.17(1.08,1.26)$ & $<0.001$ & $1.03(0.97,1.10)$ & 0.306 & $1.18(1.01,1.38)$ & 0.040 & $0.92(0.83,1.02)$ & 0.109 \\
\hline \multirow[t]{2}{*}{ Fat mass } & 1 & $1.06(1.00,1.12)$ & 0.036 & $0.98(0.93,1.02)$ & 0.291 & $1.17(1.04,1.31)$ & 0.007 & $0.91(0.84,0.98)$ & 0.014 \\
\hline & 2 & $1.12(1.06,1.20)$ & $<0.001$ & $1.00(0.95,1.05)$ & 0.891 & $1.19(1.05,1.35)$ & 0.007 & $0.91(0.84,0.99)$ & 0.032 \\
\hline \multirow[t]{2}{*}{ Hip BMD } & 1 & $1.09(1.03,1.15)$ & 0.002 & $1.02(0.98,1.07)$ & 0.331 & $1.80(1.61,2.01)$ & $<0.001$ & $0.96(0.89,1.04)$ & 0.295 \\
\hline & 2 & $1.06(0.99,1.14)$ & 0.070 & $1.06(1.00,1.12)$ & 0.049 & $2.07(1.80,2.38)$ & $<0.001$ & $1.01(0.92,1.11)$ & 0.850 \\
\hline
\end{tabular}

Table shows risk of adverse outcome per SD lower baseline level of predictor

Baseline levels ascertained at Year 2 for gait speed and at Year 1 for remaining predictors. An indicator variable for the corresponding outcomes occurring before the survival analysis follow-up was used as the stratification variable in Cox models for low trauma fracture and hospital admission; models for recurrent falls were adjusted for previous recurrent falls. Model 1: Adjusted for the four-level sex-ethnicity variable and age. Model 2: Additionally adjusted for height, weight-for-height residual (not used in models for ALM and fat mass), smoking status (ever vs never), alcohol consumption, healthy eating index, physical activity, educational attainment, home ownership, cognitive function, and number of comorbidities

$H R$ hazard ratio (odds ratios from a generalized estimating equations model are presented for recurrent falls), $S D$ standard deviation, $A L M$ appendicular lean mass, $B M D$ bone mineral density

Significant associations $(p<0.05)$ are highlighted in bold

Table 4 Muscle function, body composition, and adverse outcomes: impact of rates of loss

\begin{tabular}{|c|c|c|c|c|c|c|c|c|c|}
\hline \multirow[t]{2}{*}{ Predictor } & \multirow[t]{2}{*}{ Model } & \multicolumn{2}{|l|}{ Death } & \multicolumn{2}{|l|}{ Hospital admission } & \multicolumn{2}{|c|}{ Low trauma fracture } & \multicolumn{2}{|l|}{ Recurrent fall } \\
\hline & & $\operatorname{HR}(95 \%$ CI $)$ & $p$-value & $\operatorname{HR}(95 \% \mathrm{CI})$ & $p$-value & $\operatorname{HR}(95 \% \mathrm{CI})$ & $p$-value & OR $(95 \% \mathrm{CI})$ & $p$-value \\
\hline \multirow[t]{2}{*}{ Gait speed } & 1 & $1.21(1.14,1.28)$ & $<0.001$ & $1.11(1.06,1.16)$ & $<0.001$ & $1.03(0.93,1.14)$ & 0.601 & $1.08(1.00,1.17)$ & 0.046 \\
\hline & 2 & $1.19(1.12,1.26)$ & $<0.001$ & $1.09(1.04,1.15)$ & 0.001 & $1.01(0.91,1.13)$ & 0.818 & $1.07(0.99,1.16)$ & 0.103 \\
\hline \multirow[t]{2}{*}{ Grip strength } & 1 & $1.14(1.08,1.20)$ & $<0.001$ & $1.07(1.02,1.12)$ & 0.004 & $1.09(0.98,1.21)$ & 0.109 & $1.10(1.02,1.19)$ & 0.016 \\
\hline & 2 & $1.09(1.03,1.16)$ & 0.002 & $1.05(1.01,1.11)$ & $\mathbf{0 . 0 3 0}$ & $1.07(0.95,1.20)$ & 0.257 & $1.09(1.00,1.19)$ & 0.040 \\
\hline \multirow[t]{2}{*}{ ALM } & 1 & $1.17(1.11,1.24)$ & $<0.001$ & $1.05(1.01,1.10)$ & 0.024 & $1.06(0.95,1.19)$ & 0.279 & $1.10(1.03,1.19)$ & 0.008 \\
\hline & 2 & $1.15(1.08,1.22)$ & $<0.001$ & $1.03(0.98,1.08)$ & 0.242 & $1.06(0.94,1.20)$ & 0.345 & $1.07(0.99,1.15)$ & 0.107 \\
\hline \multirow[t]{2}{*}{ Fat mass } & 1 & $1.10(1.04,1.17)$ & 0.001 & $1.03(0.99,1.08)$ & 0.143 & $1.14(1.03,1.27)$ & 0.012 & $1.04(0.96,1.13)$ & 0.357 \\
\hline & 2 & $1.09(1.03,1.16)$ & 0.004 & $1.02(0.97,1.08)$ & 0.341 & $1.16(1.03,1.31)$ & 0.012 & $1.01(0.93,1.10)$ & 0.764 \\
\hline \multirow[t]{2}{*}{ Hip BMD } & 1 & $1.12(1.06,1.18)$ & $<0.001$ & $1.05(1.01,1.10)$ & 0.027 & $1.13(1.02,1.24)$ & 0.016 & $1.00(0.93,1.07)$ & 0.960 \\
\hline & 2 & $1.09(1.03,1.16)$ & 0.002 & $1.07(1.02,1.12)$ & 0.007 & $1.12(1.01,1.24)$ & $\mathbf{0 . 0 3 5}$ & $0.98(0.92,1.06)$ & 0.679 \\
\hline
\end{tabular}

Table shows risk of adverse outcome per SD greater rate of decline in predictor

Conditional changes (independent of baseline) were derived from Years 1 to 4 (Years 2 to 4 for gait speed and Years 1 to 3 for hip BMD). An indicator variable for the corresponding outcomes occurring before the survival analysis follow-up was used as the stratification variable in Cox models for low trauma fracture and hospital admission; models for recurrent falls were adjusted for previous recurrent falls. Model 1: Adjusted for the four-level sex-ethnicity variable and age. Model 2: Additionally adjusted for height, weight-for-height residual, smoking status (ever vs never), alcohol consumption, healthy eating index, physical activity, educational attainment, home ownership, cognitive function, and number of comorbidities

$H R$ hazard ratio (odds ratios from a generalized estimating equations model are presented for recurrent falls), $S D$ standard deviation, $A L M$ appendicular lean mass, $B M D$ bone mineral density

Significant associations $(p<0.05)$ are highlighted in bold 

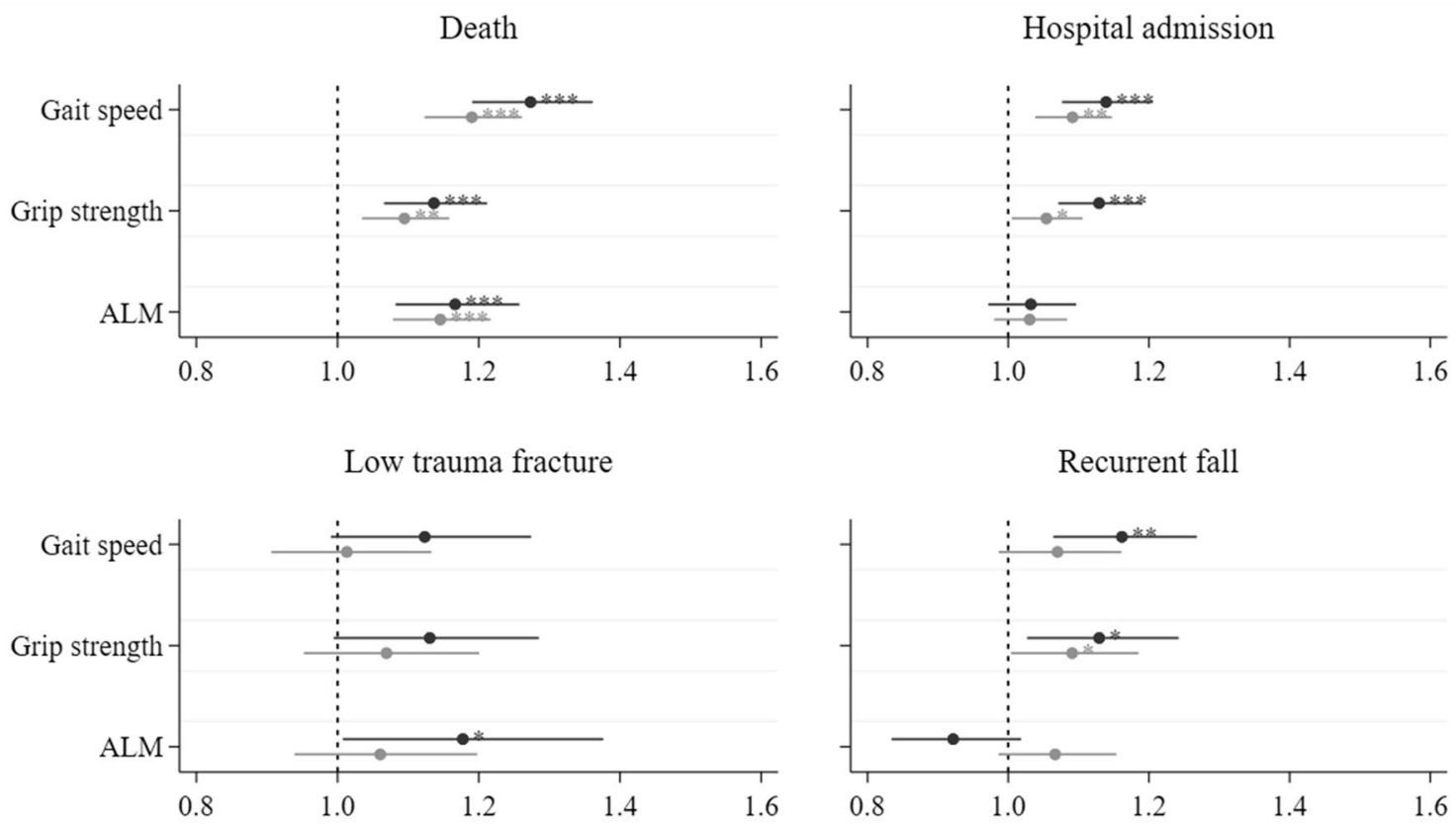

Hazard ratio $(95 \% \mathrm{CI})$

$* \mathrm{p}<0.05, * * \mathrm{p}<0.01, * * * \mathrm{p}<0.001$

- Lower level Greater decline

Fig. 1 Risk of adverse outcomes per SD lower baseline level and per SD greater decline in sarcopenia component. $H R$ hazard ratio (odds ratios from a generalized estimating equations model are presented for recurrent falls), $S D$ standard deviation, $A L M$ appendicular lean mass. Baseline levels ascertained at Year 2 for gait speed and at Year 1 for remaining predictors. Conditional changes (independent of baseline) were derived from Years 1 to 4 (Years 2 to 4 for gait speed). An indicator variable for the corresponding outcomes occurring before

\section{Sensitivity Analyses}

Determinants of greater numbers of hospital admissions, both after adjustment for sex, ethnicity and age and in fullyadjusted analysis, were lower gait speed, grip strength, and hip BMD and greater declines in gait speed (data not shown). Descriptive statistics for competing risk analyses are presented in eTable 1 (Online Resource). Some associations for hospital admission and low trauma fracture were attenuated when investigated using competing risk models (eTable 2 in Online Resource); however, some key findings such as relationships between lower gait speed and grip strength in relation to increased risk of hospital admission and regarding lower fat mass and hip BMD in relation to greater risk of low trauma fracture were robust in these sensitivity analyses. Baseline levels and changes in parameters were most strongly associated with underlying causes of mortality that were not cardiovascular- or cancerrelated, followed by cardiovascular-related mortality and then cancer-related mortality (eTables 3, 4 and 5 in Online the survival analysis follow-up was used as the stratification variable in Cox models for low trauma fracture and hospital admission; models for recurrent falls were adjusted for previous recurrent falls. Models were adjusted for the four-level sex-ethnicity variable, age, height, weight-for-height residual (not used in models for level relating to ALM), smoking status (ever vs never), alcohol consumption, healthy eating index, physical activity, educational attainment, home ownership, cognitive function, and number of comorbidities

Resource). Results for sensitivity analyses relating to ALM (use of ALM residuals and adjusting changes in ALM for changes in body weight) were similar to those from the main analyses (data not shown).

\section{Discussion}

Among participants of the Health ABC Study, we have examined baseline levels and changes in sarcopenia components in relation to rates of mortality, hospital admission, low trauma fracture, and recurrent falls over a subsequent follow-up period ranging from 10 to 14 years. Lower gait speed and grip strength were associated with increased rates of mortality, hospital admission, and recurrent falls; declines in these parameters were related to increased rates of mortality and hospital admission. In contrast, robust relationships regarding levels and changes in ALM were only observed in relation to mortality. 

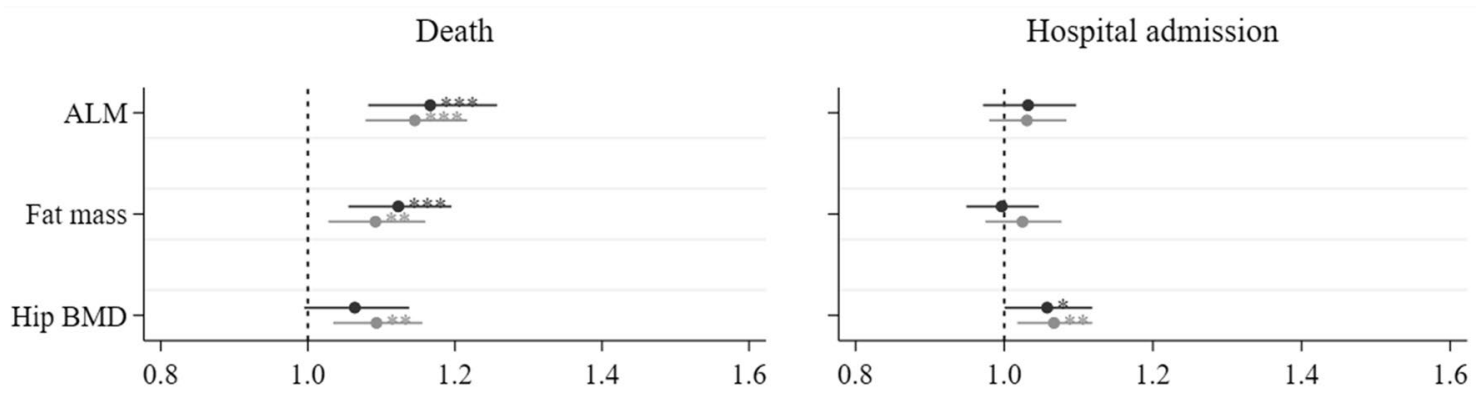

Low trauma fracture

Recurrent fall
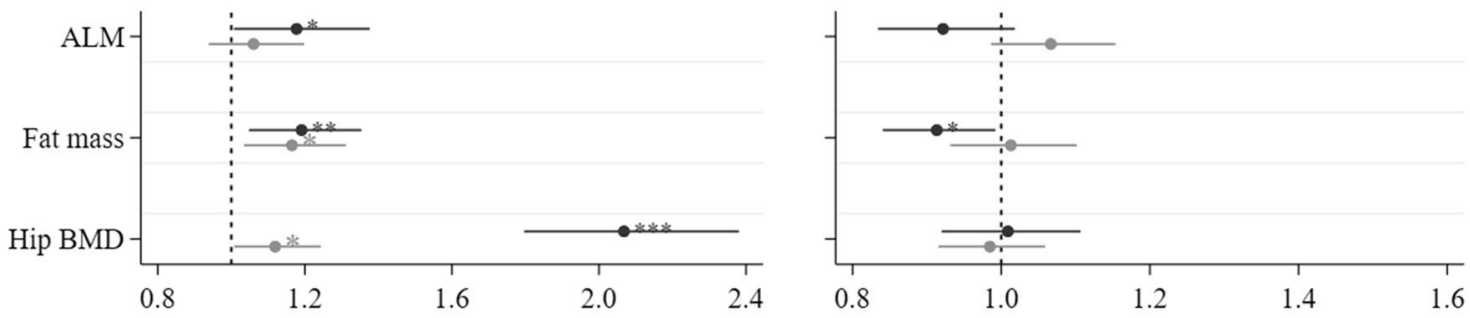

Hazard ratio $(95 \% \mathrm{CI})$

$* \mathrm{p}<0.05, * * \mathrm{p}<0.01, * * * \mathrm{p}<0.001$

- Lower level Greater decline

Fig. 2 Risk of adverse outcomes per SD lower baseline level and per $\mathrm{SD}$ greater decline in body composition component. $H R$ hazard ratio (odds ratios from a generalized estimating equations model are presented for recurrent falls), $S D$ standard deviation, $A L M$ appendicular lean mass, $B M D$ bone mineral density. Baseline levels ascertained at Year 1. Conditional changes (independent of baseline) were derived from Years 1 to 4 (Years 1 to 3 for hip BMD). An indicator variable for the corresponding outcomes occurring before the survival analy-

The magnitude of associations differed for the baseline level and change exposures. For baseline levels and changes in gait speed and grip strength in relation to mortality and hospital admission, effect sizes for baseline levels were higher than the corresponding effect sizes for rates of change (Fig. 1). Regarding mortality, hazard ratios were greater for baseline levels of gait speed compared to grip strength (fully-adjusted hazard ratios with 95\% CI 1.27 [1.19, 1.36] vs $1.14[1.07,1.21])$ whereas these were similar for hospital admission (gait speed: 1.14 [1.08, 1.21], grip strength 1.13 $[1.07,1.19])$. The relative contribution of grip strength level and change for explaining variation in rates of mortality was similar; the relative contribution was higher for gait speed level compared to gait speed change and lower for ALM level compared to ALM change.

Previous studies have examined levels and changes in gait speed, grip strength and ALM in relation to risk of mortality. The relationships between lower levels and greater declines in grip strength and increased mortality risk are widely established [4, 32-35]. Furthermore, previous literature has sis follow-up was used as the stratification variable in Cox models for low trauma fracture and hospital admission; models for recurrent falls were adjusted for previous recurrent falls. Models were adjusted for the four-level sex-ethnicity variable, age, height, weight-for-height residual (not used in models for level relating to ALM and fat mass), smoking status (ever vs never), alcohol consumption, healthy eating index, physical activity, educational attainment, home ownership, cognitive function, and number of comorbidities

established low gait speed [5, 36, 37] and greater declines in gait speed [6, 38] as risk factors for mortality. The relationship between lower ALM and increased mortality risk was reported in the Cardiovascular Health Study [39], and greater declines in ALM in relation to higher risk of mortality were found in the MINOS study, comprising older men [40], and in a previous Health ABC analysis over a shorter follow-up duration [31]; the associations reported in the previous Health $\mathrm{ABC}$ analysis were not robust to adjustment for changes in weight.

Levels and changes in these parameters in relation to risk of hospital admission, fractures, and falls have also been explored previously. Lower grip strength was related to increased risk of osteoporotic fracture in a meta-analysis of the MrOS Study [41]; was related to risk of hospital admission in a previous analysis of the Health ABC Study over a shorter follow-up duration of 5 years [23]; and was predictive of emergency and long-stay hospital admission among men and women from the Hertfordshire Cohort Study [42]. Similar to our findings, upper extremity weakness was 
associated with increased risk of falls and recurrent falls in a systematic review and meta-analysis [43], and rate of grip strength decline was related to subsequent falls in the Women's Health and Aging Study (WHAS) II [44].

Similarly to our findings, slower gait speed was related to greater risk of hospital admission in a previous analysis of the Health ABC Study over a shorter follow-up [23] and future falls in the Einstein Aging Study [45]. In contrast to our findings, slower gait speed was a risk factor for osteoporotic fracture among men in MrOS [41], and gait speed decline was related to risk of incident falls and hip fracture in the MOBILIZE Boston Study [46] and in the Study of Osteoporotic Fractures (SOF) [47], respectively.

Robust relationships between ALM levels and outcomes other than mortality were not found in our study. This is in agreement with a previous analysis of the Health ABC Study where lean mass was not related to risk of hospitalization over a shorter follow-up [23]. Findings which differ from our study include associations between lower ALM index and greater risk of fragility fracture among women from the Os des Femmes de Lyon (OFELY) study [48] and major osteoporotic fracture among men in MrOS [41]; lower ALM was only related to higher risk of low trauma fracture after adjustment for potential confounders in our study.

There are several potential mechanisms which may relate impairment in sarcopenia components to increased risk of mortality. Deficits in components of sarcopenia are correlated with low socio-economic position, poor health behaviors, and increased comorbidity [28] which are established risk factors for mortality. However, associations in this study between muscle mass, strength, and function and mortality remain after adjustment for these factors, suggesting that they do not fully explain the associations observed. Another possibility is that physiological processes such as increased oxidative stress, inflammation, and endocrine dysfunction are contributing to age-related declines in sarcopenia components as well as increased mortality risk [36]. As well as sufficient strength, walking speed also requires motor control and involves multiple anatomical systems; low gait speed may therefore reflect impairment in these systems, leading to greater risk of mortality [5].

Overall our findings are broadly in agreement with the published literature; possible reasons for discrepancies may be due to differences relating to outcomes (such as use of recurrent falls as opposed to incident falls), adjustments, methods used to derive change measures, and follow-up times. However, a key strength of this study is the inclusion of a wide range of musculoskeletal parameters and adverse health outcomes in a single, well-characterized cohort. This enables a comparison of the magnitude of associations between baseline levels and rates of decline in these parameters in relation to clinical outcomes. In contrast, the comparability of associations from previous literature may be reduced due to differences in age ranges and nationalities of participants between studies. Although there are limitations of deriving changes using data from two time-points [49], strengths of the statistical approach implemented include the use of conditional changes in parameters that have zero correlation with baseline levels; a long survival analysis follow-up time (10-14 years) occurring after measurement of exposures; and sensitivity analyses for hospital admission and low trauma fracture which account for the competing risk of death.

A limitation of this study is that participants had no mobility disability at baseline. This limits the generalizability of the findings to the wider population of communitydwelling older people in this age range. Similarly, the exclusion of grip strength values from participants with severe hand pain or recent surgery may have introduced bias as weak grip strength is an established risk factor for adverse health outcomes. In addition, the analyses were restricted to participants with both baseline and change measures for at least one of the parameters of interest, meaning that participants who died before Year 4 (Year 3 for hip BMD) were excluded from the analysis, resulting in attrition bias. These limitations may have led to an underestimation in the magnitude of the reported associations. However, our analyses were internal to this sample; substantial bias should only have occurred if the associations examined differed markedly between Health $\mathrm{ABC}$ participants who were and were not included in the analysis; this seems unlikely. Finally, as in all observational studies, there is the possibility that the associations observed may be explained by residual confounding.

Our findings have important clinical implications. The strong associations between slower gait speed and weaker grip strength and multiple adverse health outcomes concur with the position of the Sarcopenia Definitions and Outcomes Consortium (SDOC) that these components, rather than lean mass, should be included in a definition of sarcopenia due to their greater capacity to predict clinically relevant outcomes. For the same reason, the revised definition of the European Working Group on Sarcopenia in Older People (EWGSOP2) regards low muscle strength, rather than lean mass, as the primary component of sarcopenia. Lower baseline values and greater declines in gait speed and grip strength were related to increased rates of mortality and hospital admission in our study. Therefore, interventions aimed at maximizing levels attained in earlier adult life as well as reducing rates of decline from midlife onwards may reduce the burden of disease in this age group as well as improving musculoskeletal health in older age. There is evidence that some musculoskeletal parameters, such as grip strength, have differential determinants for level and change [50] so a focus on both sets of determinants is important for intervention strategies. 


\section{Conclusion}

In this study, both lower levels and greater declines in gait speed and grip strength were associated with mortality and hospital admission, with larger effect sizes for levels than rates of change. This suggests that the combined use of absolute levels of parameters as well as rates of change over time could be used to improve the identification of individuals most at risk of adverse outcomes and who are likely to benefit most from interventions.

Acknowledgements This work was supported by the National Institute on Aging (NIA) Grants (R01AG027017, P30AG024827, T32AG021885, and K07AG033174); the Intramural Research program of the National Institutes of Health (N01AG62101, N01AG62103, N01AG62106, and R01AG028050); a National Institute of Nursing Research Grant (R01NR012459); the Medical Research Council (MRC_MC_UU_12011/2 and MRC_MC_UP_A620_1015) and the University of Southampton, UK. Dr Fielding's participation was supported by the U.S. Department of Agriculture (USDA), under agreement No. 58-1950-4-003. Any opinions, findings, conclusions, or recommendations expressed in this publication are those of the authors and do not necessarily reflect the view of the USDA. The authors thank the participants of the Health, Aging, and Body Composition Study as well as members of the scientific and data collection teams.

Author Contributions LW conducted the statistical analysis and wrote the first draft of the manuscript; HES provided guidance regarding the statistical analysis; EMD and CC designed the study; JAC, EJS, and $A B N$ were investigators of the Health ABC Study. All authors made substantial contributions to the manuscript and approved the final version.

\section{Compliance with Ethical Standards}

Conflict of interest Cyrus Cooper reports personal fees (outside the submitted work) from Amgen, Danone, Eli Lilly, GSK, Kyowa Kirin, Medtronic, Merck, Nestle, Novartis, Pfizer, Roche, Servier, Shire, Takeda, and UCB. Elaine M. Dennison reports personal fees (outside the submitted work) from Pfizer Healthcare and from the UCB Discussion panel. Nicholas C. Harvey reports consultancy, lecture fees, and honoraria (outside the submitted work) from Alliance for Better Bone Health, AMGEN, MSD, Eli Lilly, Servier, Shire, UCB, Kyowa Kirin, Consilient Healthcare, Radius Health, and Internis Pharma. Roger A. Fielding reports grants from the National Institutes of Health (National Institute on Aging) and the USDA, during the conduct of the study; grants, personal fees, and other from Axcella Health, other from Inside Tracker, grants and personal fees from Biophytis, grants and personal fees from Astellas, personal fees from Cytokinetics, personal fees from Amazentis, grants and personal fees from Nestle', personal fees from Glaxo Smith Kline, outside the submitted work. Leo D. Westbury, Holly E. Syddall, Nicholas R. Fuggle, Jane A. Cauley, Eric J. Shiroma, and Anne B. Newman declare that they have no conflicts of interest.

Ethical Approval The study was approved by the institutional review boards at the University of Tennessee and the University of Pittsburgh.

Human and Animal Rights All procedures performed in studies involving human participants were in accordance with the ethical standards of the institutional and/or national research committee and with the 1964 Helsinki declaration and its later amendments or comparable ethical standards.
Informed Consent Written informed consent was provided by all participants.

Open Access This article is licensed under a Creative Commons Attribution 4.0 International License, which permits use, sharing, adaptation, distribution and reproduction in any medium or format, as long as you give appropriate credit to the original author(s) and the source, provide a link to the Creative Commons licence, and indicate if changes were made. The images or other third party material in this article are included in the article's Creative Commons licence, unless indicated otherwise in a credit line to the material. If material is not included in the article's Creative Commons licence and your intended use is not permitted by statutory regulation or exceeds the permitted use, you will need to obtain permission directly from the copyright holder. To view a copy of this licence, visit http://creativecommons.org/licenses/by/4.0/.

\section{References}

1. Beaudart C, Rizzoli R, Bruyère O, Reginster J-Y, Biver E (2014) Sarcopenia: burden and challenges for public health. Arch Public Health 72:45

2. Cruz-Jentoft AJ, Bahat $\mathrm{G}$, Bauer $\mathrm{J}$, Boirie $\mathrm{Y}$, Bruyère $\mathrm{O}$, Cederholm T, Cooper C, Landi F, Rolland Y, Sayer AA (2018) Sarcopenia: revised European consensus on definition and diagnosis. Age Ageing 48:16-31

3. Bhasin S, Travison TG, Manini TM, Patel S, Pencina KM, Fielding RA, Magaziner JM, Newman AB, Kiel DP, Cooper C (2020) Sarcopenia definition: the position statements of the sarcopenia definition and outcomes consortium. J Am Geriatr Soc 68:1410-1418

4. Prasitsiriphon O, Pothisiri W (2018) Associations of grip strength and change in grip strength with all-cause and cardiovascular mortality in a European older population. Clin Med Insights Cardiol 12:1179546818771894

5. Studenski S, Perera S, Patel K, Rosano C, Faulkner K, Inzitari M, Brach J, Chandler J, Cawthon P, Connor EB, Nevitt M, Visser M, Kritchevsky S, Badinelli S, Harris T, Newman AB, Cauley J, Ferrucci L, Guralnik JM (2011) Gait speed and survival in older adults. JAMA 305:50-58

6. White DK, Neogi T, Nevitt MC, Peloquin CE, Zhu Y, Boudreau RM, Cauley JA, Ferrucci L, Harris TB, Satterfield SM, Simonsick EM, Strotmeyer ES, Zhang Y (2013) Trajectories of gait speed predict mortality in well-functioning older adults: the Health, Aging and Body Composition study. J Gerontol A Biol Sci Med Sci 68:456-464

7. Brown JC, Harhay MO, Harhay MN (2016) Sarcopenia and mortality among a population-based sample of community-dwelling older adults. J Cachexia Sarcopenia Muscle 7:290-298

8. Bianchi L, Ferrucci L, Cherubini A, Maggio M, Bandinelli S, Savino E, Brombo G, Zuliani G, Guralnik JM, Landi F, Volpato S (2016) The predictive value of the EWGSOP definition of sarcopenia: results from the InCHIANTI study. J Gerontol A Biol Sci Med Sci 71:259-264

9. Yu R, Leung J, Woo J (2014) Incremental predictive value of sarcopenia for incident fracture in an elderly Chinese cohort: results from the Osteoporotic Fractures in Men (MrOs) Study. J Am Med Dir Assoc 15:551-558

10. Scott D, Johansson J, McMillan LB, Ebeling PR, Nordstrom P, Nordstrom A (2019) Associations of sarcopenia and its components with bone structure and incident falls in Swedish older adults. Calcif Tissue Int 105:26-36 
11. Houston DK, Nicklas BJ, Ding J, Harris TB, Tylavsky FA, Newman AB, Lee JS, Sahyoun NR, Visser M, Kritchevsky SB (2008) Dietary protein intake is associated with lean mass change in older, community-dwelling adults: the Health, Aging, and Body Composition (Health ABC) Study. Am J Clin Nutr 87:150-155

12. National Institute on Aging (2020) Introducing the health $A B C$ study: the dynamics of health, aging, and body composition. https ://www.healthabc.nia.nih.gov/. Accessed 16 Oct 2020

13. National Institute on Aging (2020) Health ABC operations manual volume III: grip strength hand-held dynamometry. https://healt habc.nia.nih.gov/sites/default/files/grip.om1_0.pdf. Accessed 16 Oct 2020

14. Visser M, Fuerst T, Lang T, Salamone L, Harris TB (1999) Validity of fan-beam dual-energy X-ray absorptiometry for measuring fat-free mass and leg muscle mass. Health, aging, and body composition study-dual-energy X-ray absorptiometry and body composition working group. J Appl Physiol 87:1513-1520

15. Salamone LM, Fuerst T, Visser M, Kern M, Lang T, Dockrell M, Cauley JA, Nevitt M, Tylavsky F, Lohman TG (2000) Measurement of fat mass using DEXA: a validation study in elderly adults. J Appl Physiol 89:345-352

16. Ainsworth BE, Haskell WL, Leon AS, Jacobs JD, Montoye HJ, Sallis JF, Paffenbarger JR (1993) Compendium of physical activities: classification of energy costs of human physical activities. Med Sci Sports Exerc 25:71-80

17. Visser M, Simonsick EM, Colbert LH, Brach J, Rubin SM, Kritchevsky SB, Newman AB, Harris TB (2005) Type and intensity of activity and risk of mobility limitation: the mediating role of muscle parameters. J Am Geriatr Soc 53:762-770

18. Goldman SE, Stone KL, Ancoli-Israel S, Blackwell T, Ewing SK, Boudreau R, Cauley JA, Hall M, Matthews KA, Newman AB (2007) Poor sleep is associated with poorer physical performance and greater functional limitations in older women. Sleep 30:1317-1326

19. Westbury LD, Syddall HE, Fuggle NR, Dennison EM, Cauley JA, Shiroma EJ, Fielding RA, Newman AB, Cooper C (2020) Long-term rates of change in musculoskeletal aging and body composition: findings from the Health, Aging and Body Composition Study. Calcif Tissue Int 106:616-624

20. Teng E, Chui H (1987) The modified mini-mental state examination (3MS). Can J Psychiatry 41:114-121

21. Rosano C, Simonsick EM, Harris TB, Kritchevsky SB, Brach J, Visser M, Yaffe K, Newman AB (2005) Association between physical and cognitive function in healthy elderly: the health, aging and body composition study. Neuroepidemiology 24:8-14

22. Kennedy E, Ohls J, Carlson S, Fleming K (1995) The Healthy Eating Index: design and applications. J Am Diet Assoc 95:1103-1108

23. Cawthon PM, Fox KM, Gandra SR, Delmonico MJ, Chiou CF, Anthony MS, Sewall A, Goodpaster B, Satterfield S, Cummings SR, Harris TB (2009) Do muscle mass, muscle density, strength, and physical function similarly influence risk of hospitalization in older adults? J Am Geriatr Soc 57:1411-1419

24. Yenchek RH, Ix JH, Shlipak MG, Bauer DC, Rianon NJ, Kritchevsky SB, Harris TB, Newman AB, Cauley JA, Fried LF (2012) Bone mineral density and fracture risk in older individuals with CKD. Clin J Am Soc Nephrol 7:1130-1136

25. Marcum ZA, Perera S, Newman AB, Thorpe JM, Switzer GE, Gray SL, Simonsick EM, Shorr RI, Bauer DC, Castle NG, Studenski SA, Hanlon JT (2015) Antihypertensive use and recurrent falls in community-dwelling older adults: findings from the health ABC study. J Gerontol A Biol Sci Med Sci 70:1562-1568

26. Marcum ZA, Perera S, Thorpe JM, Switzer GE, Castle NG, Strotmeyer ES, Simonsick EM, Ayonayon HN, Phillips CL, Rubin S, Zucker-Levin AR, Bauer DC, Shorr RI, Kang Y, Gray SL, Hanlon JT (2016) Antidepressant use and recurrent falls in community-dwelling older adults: findings from the health $\mathrm{ABC}$ study. Ann Pharmacother 50:525-533

27. Marcum ZA, Perera S, Thorpe JM, Switzer GE, Gray SL, Castle NG, Strotmeyer ES, Simonsick EM, Bauer DC, Shorr RI, Studenski SA, Hanlon JT (2015) Anticholinergic use and recurrent falls in community-dwelling older adults: findings from the health ABC study. Ann Pharmacother 49:1214-1221

28. Curtis E, Litwic A, Cooper C, Dennison E (2015) Determinants of muscle and bone aging. J Cell Physiol 230:2618-2625

29. Fine JP, Gray RJ (1999) A proportional hazards model for the subdistribution of a competing risk. J Am Stat Assoc 94:496-509

30. Newman AB, Kupelian V, Visser M, Simonsick E, Goodpaster B, Nevitt M, Kritchevsky SB, Tylavsky FA, Rubin SM, Harris TB (2003) Sarcopenia: alternative definitions and associations with lower extremity function. J Am Geriatr Soc 51:1602-1609

31. Santanasto AJ, Goodpaster BH, Kritchevsky SB, Miljkovic I, Satterfield S, Schwartz AV, Cummings SR, Boudreau RM, Harris TB, Newman AB (2017) Body composition remodeling and mortality: the health aging and body composition study. J Gerontol A Biol Sci Med Sci 72:513-519

32. Syddall HE, Westbury LD, Dodds R, Dennison E, Cooper C, Sayer AA (2017) Mortality in the hertfordshire ageing study: association with level and loss of hand grip strength in later life. Age Ageing 46:407-412

33. Ling CHY, Taekema D, De Craen AJM, Gussekloo J, Westendorp RGJ, Maier AB (2010) Handgrip strength and mortality in the oldest old population: the Leiden 85-plus study. CMAJ 182:429-435

34. Granic A, Davies K, Jagger CM, Dodds R, Kirkwood TB, Sayer AA (2017) Initial level and rate of change in grip strength predict all-cause mortality in very old adults. Age Ageing 46:970-976

35. Metter EJ, Talbot LA, Schrager M, Conwit R (2002) Skeletal muscle strength as a predictor of all-cause mortality in healthy men. J Gerontol A Biol Sci Med Sci 57:B359-B365

36. Cooper R, Kuh D, Hardy R (2010) Objectively measured physical capability levels and mortality: systematic review and metaanalysis. BMJ 341:c4467

37. Dumurgier J, Elbaz A, Ducimetière P, Tavernier B, Alpérovitch A, Tzourio C (2009) Slow walking speed and cardiovascular death in well functioning older adults: prospective cohort study. BMJ 339:b4460

38. Sabia S, Dumurgier J, Tavernier B, Head J, Tzourio C, Elbaz A (2013) Change in fast walking speed preceding death: results from a prospective longitudinal cohort study. J Gerontol A Biol Sci Med Sci 69:354-362

39. Spahillari A, Mukamal K, DeFilippi C, Kizer JR, Gottdiener JS, Djoussé L, Lyles MF, Bartz TM, Murthy VL, Shah RV (2016) The association of lean and fat mass with all-cause mortality in older adults: the cardiovascular health study. Nutr Metab Cardiovasc Dis 26:1039-1047

40. Szulc P, Munoz F, Marchand F, Chapurlat R, Delmas PD (2010) Rapid loss of appendicular skeletal muscle mass is associated with higher all-cause mortality in older men: the prospective MINOS study. Am J Clin Nutr 91:1227-1236

41. Harvey NC, Odén A, Orwoll E, Lapidus J, Kwok T, Karlsson MK, Rosengren BE, Ribom E, Cooper C, Cawthon PM (2018) Measures of physical performance and muscle strength as predictors of fracture risk independent of FRAX, falls, and aBMD: a meta-analysis of the osteoporotic fractures in men (MrOS) study. J Bone Miner Res 33:2150-2157

42. Simmonds SJ, Syddall HE, Westbury LD, Dodds RM, Cooper C, Aihie Sayer A (2015) Grip strength among community-dwelling older people predicts hospital admission during the following decade. Age Ageing 44:954-959 
43. Moreland JD, Richardson JA, Goldsmith CH, Clase CM (2004) Muscle weakness and falls in older adults: a systematic review and meta-analysis. J Am Geriatr Soc 52:1121-1129

44. Xue Q-L, Walston JD, Fried LP, Beamer BA (2011) Prediction of risk of falling, physical disability, and frailty by rate of decline in grip strength: the women's health and aging study. Arch Intern Med 171:1119-1121

45. Verghese J, Holtzer R, Lipton RB, Wang C (2009) Quantitative gait markers and incident fall risk in older adults. J Gerontol A Biol Sci Med Sci 64:896-901

46. Quach L, Galica AM, Jones RN, Procter-Gray E, Manor B, Hannan MT, Lipsitz LA (2011) The nonlinear relationship between gait speed and falls: the maintenance of balance, independent living, intellect, and zest in the elderly of Boston study. J Am Geriatr Soc 59:1069-1073

47. Barbour KE, Lui L-Y, McCulloch CE, Ensrud KE, Cawthon PM, Yaffe K, Barnes DE, Fredman L, Newman AB, Cummings SR (2016) Trajectories of lower extremity physical performance: effects on fractures and mortality in older women. J Gerontol A Biol Sci Med Sci 71:1609-1615

48. Sornay-Rendu E, Duboeuf F, Boutroy S, Chapurlat R (2017) Muscle mass is associated with incident fracture in postmenopausal women: The OFELY study. Bone 94:108-113

49. Singer JD, Willett JB, Willett JB (2003) Applied longitudinal data analysis: modeling change and event occurrence. Oxford University Press, Oxford

50. Syddall HE, Westbury L, Shaw S, Dennison E, Cooper C, Gale C (2018) Correlates of level and loss of grip strength in later life: findings from the English longitudinal study of ageing and the hertfordshire cohort study. Calcif Tissue Int 102:53-63

Publisher's Note Springer Nature remains neutral with regard to jurisdictional claims in published maps and institutional affiliations. 